\title{
Features and outcomes of immunoglobulin therapy in patients with Good syndrome at Thailand's largest tertiary referral hospital
}

\author{
Torpong Thongngarm, ${ }^{1}$ Adhiratha Boonyasiri, ${ }^{2}$ Panitan Pradubpongsa, ${ }^{3}$ Nattaporn Tesavibul, ${ }^{4}$ Tauangtham Anekpuritanang, \\ Piyawut Kreetapirom, ${ }^{6}$ Mongkhon Sompornrattanaphan ${ }^{1}$
}

\begin{abstract}
Background: Good syndrome (GS) is an adult-onset immunodeficiency characterized by coexisting thymoma and hypogammaglobulinemia. Clinical course after treatment with intravenous immunoglobulin (IVIg) has rarely been reported.

Objective: To investigate and report the clinical course and outcomes of GS patients after treatment with IVIg at Thailand's largest national tertiary referral hospital

Methods: This retrospective chart review included patients diagnosed with GS and treated with IVIg during the 1 January 2005 to 31 December 2015 study period.

Results: Nine GS patients with a median age at diagnosis of 53 years were included. Pneumonia and sepsis were the most common clinical manifestations. Six infectious organisms suggestive of cell-mediated immunity defect occurred in six patients, including cytomegalovirus (CMV), Mycobacterium tuberculosis, Mycobacterium abscessus, Herpes simplex virus (HSV), Pneumocystis jirovecii, and Aspergillus. Mean serum IgG level was $317 \mathrm{mg} / \mathrm{dL}$. Eight patients had very low to undetectable B-cells. Five patients had either low CD4 number or impaired T-cell function, and one patient had both. All patients received IVIg replacement therapy monthly at a dose of $0.4 \mathrm{~g} / \mathrm{kg}$. The mean trough IgG level was $881 \mathrm{mg} / \mathrm{dL}$. After treatment with IVIg replacement, seven patients had favorable clinical outcomes. However, two patients expired due to septicemia.

Conclusion: Clinical outcomes of patients with GS are more dependent on the severity of infections and associated hematologic and autoimmune diseases than on the severity of thymoma itself. Therefore, early recognition and prompt IVIg replacement may change the natural course of this condition and may be successful in keeping the patient infections free.
\end{abstract}

Keywords: Thymoma, hypogammaglobulinemia, immunodeficiency, infection, intravenous immunoglobulin

\section{From:}

${ }^{1}$ Division of Allergy and Clinical Immunology, Department of Medicine, Faculty of Medicine Siriraj Hospital, Mahidol University, Bangkok, Thailand

${ }^{2}$ Division of Clinical Epidemiology, Department of Research and Development, Faculty of Medicine Siriraj Hospital,

Mahidol University, Bangkok, Thailand

Division of Allergy and Clinical Immunology, Department of Medicine, Phramongkutklao Hospital, Bangkok, Thailand

${ }^{4}$ Department of Ophthalmology, Faculty of Medicine Siriraj Hospital, Mahidol University, Bangkok, Thailand

Department of Pathology, Faculty of Medicine Siriraj Hospital, Mahidol University, Bangkok, Thailand

${ }^{6}$ Division of Pediatrics, Chaophya Hospital, Bangkok, Thailand

\section{Corresponding author:}

Mongkhon Sompornrattanaphan

Division of Allergy and Clinical Immunology, Department of Medicine

Faculty of Medicine Siriraj Hospital, Mahidol University

2 Prannok Road, Bangkoknoi

Bangkok 10700, Thailand

Email: mongkhon.som@mahidol.ac.th

\section{Introduction}

Good syndrome (GS) was first reported by Robert Good in 1954. This syndrome is an adult-onset immunodeficiency characterized by thymoma and hypogammaglobulinemia which usually presents in the fourth to fifth decade of life. ${ }^{1} \mathrm{Im}$ munological abnormalities in GS include decrease of peripheral B-cells, hypogammaglobulinemia, variable cell-mediated 
immunity (CMI) defect, CD4 lymphopenia, inverted CD4/CD8 ratio and impaired T-cell functions. ${ }^{2}$ The pathogenesis of GS is not completely understood. At least three hypotheses had been proposed. First, an interferon-like cytokine from bone marrow stromal cell line causing an arrest in growth and differentiation of B cells. Second, the loss of memory or naïve CD4 $\mathrm{T}$ cells causing a CMI defect. Third, T cell or autoantibodies causing an autoimmune process as a consequence of thymoma. ${ }^{3}$

Sinopulmonary infections by Haemophilus influenzae and Pseudomonas aeruginosa are the most common manifestations. Opportunistic infections by cytomegalovirus (CMV) or Candida can also develop. Associated autoimmune diseases, including pure red cell aplasia (PRCA), myasthenia gravis (MG), oral lichen planus, aplastic anemia and primary sclerosing cholangitis had been reported. ${ }^{4-6}$ Regular administration of intravenous immunoglobulin (IVIg) is the mainstay of therapy to prevent infections but cannot restore immunological abnormalities. Moreover, 5- and 10-year survival rates of GS patients were reported to be $75 \%$ and $33 \%$, respectively which found to be lower compared with patients with common variable immunodeficiency (CVID). ${ }^{5}$ Immunological problems other than hypogammaglobulinemia might involve in the course of disease. Systematic review in the clinical features and laboratory findings of GS cases was recently published. ${ }^{7,8}$ IVIG replacement was only reported to have a favorable outcome in controlling and preventing the infection in a literature review. ${ }^{8}$

The aim of this study was to investigate and report the clinical characteristics and outcomes of GS patients after treatment with IVIg at Thailand's largest national tertiary referral hospital.

\section{Methods}

This retrospective chart review included patients diagnosed with GS treated with IVIg at Division of Allergy and Clinical Immunology, Department of Medicine, Faculty of Medicine Siriraj Hospital, Mahidol University during the January 2005 to December 2015. In this study, GS or thymoma-associated immunodeficiency was defined as the simultaneous presence of immunodeficiency, thymoma, and hypogammaglobulinemia. Anti-HIV testing using enzyme-linked immunosorbent assay (ELISA) technique was negative in all patients. Medical records were reviewed to collect data regarding thymoma, immunologic disorders, infections, associated autoimmune diseases, neoplastic diseases and outcome after treatment with regular IVIg replacement. Immunologic studies were performed at our center's accredited immunology laboratory, Department of Immunology, Faculty of Medicine Siriraj Hospital, Mahidol University. Quantitative immunoglobulin levels (IgG, IgA, and IgM) were measured by automated nephelometry (BN ProSpec ${ }^{\circledast}$ System; Siemens Healthcare GmbH, Erlangen, Germany). CD4, CD8, B cell (CD19), and NK cell (CD16/56) number determination was performed by direct immunofluorescence and then measured by flow cytometry. In order to examine T-cell function, lymphoproliferation was performed by stimulating $\mathrm{T}$-cell with phytohemagglutinin (PHA), after which ${ }^{3} \mathrm{H}$-thymidine uptake measurement was used to evaluate any increase in T-cell.

The protocol for this study was approved by the Siriraj Institutional Review Board (SIRB), Faculty of Medicine Siriraj Hospital, Mahidol University, Bangkok, Thailand (approval no. $562 / 2557)$.

\section{Results}

Nine patients met the diagnostic criteria for GS and were included. The clinical characteristics before and after IVIG replacement were summarized in table 1. Median age at diagnosis was 53 years for GS and 52 years for thymoma. The median interval in the diagnosis of GS after detection of thymoma was one year (4-19 months). Eight cases were initially presented with thymoma before the diagnosis of GS. Only one case (patients 7) presented with severe infection before the recognition of thymoma. Eight of nine patients had undergone thymectomy, and four of those received radiotherapy. Immunologic profiles of nine patients were summarized in table 2. The mean IgG level was $317 \mathrm{mg} / \mathrm{dL}$. Five patients had panhypogammaglobulinemia. Eight patients had very low to undetectable B-cells. Three patients had low number of CD4 cells and six patients had decreased CD4/CD8 ratio. Four patients had impaired T-cell function. However, three of those still had a normal number of CD4 cells. Six patients had decreased NK cells.

Pneumonia and sepsis were the most common manifestations. Six pathogens suggestive of CMI defect occurred in six patients, including CMV, Mycobacterium tuberculosis, Mycobacterium abscessus, Herpes simplex virus (HSV), Pneumocystis jirovecii, and Aspergillus. Seven patients had already developed bronchiectasis by the time GS was diagnosed. Chronic diarrhea was found in one patient. Colonic tissue revealed unspecified chronic inflammation without evidences of infection, granuloma or neoplasm. Six patients had autoimmune diseases, including $\mathrm{MG}$ and PRCA. All patients received monthly IVIg replacement, with a mean dose of $0.4 \mathrm{~g} / \mathrm{kg}$. The mean trough $\mathrm{IgG}$ level was $881 \mathrm{mg} / \mathrm{dL}$. Median duration of follow-up was two years. Seven patients clinically improved with less frequent episodes of infection. Two patients died after the diagnosis of GS despite achieving trough IgG level from IVIg replacement.

Authors have illustrated two cases with interesting clinical presentations and histopathologic findings, including patient 7 and patient 4. Patient 7, previously healthy, presented with prolonged fever and generalized lymphadenopathy. He had previously been treated with anti-tuberculosis medications for several months without improvement because of a pathological finding of caseating granulomatous lymphadenitis. Computed tomography (CT) of the chest revealed heterogeneous enhancing soft tissue mass in anterior mediastinum $2.6 \times 1.5 \mathrm{~cm}$ in size, consistent with thymoma. Multiple calcified lymph nodes at prevascular, bilateral paratracheal, subcarinal, paraaortic, subaortic, bilateral hilar and left supraclavicular regions were detected, and multiple patchy consolidations scattering at both lungs were noted. Pathological result from cervical lymph node morphologically consistent with Pneumocystis spp. (figure 1). The molecular diagnosis using sequence analysis of the fungal internal transcribed spacer (ITS) region amplification 


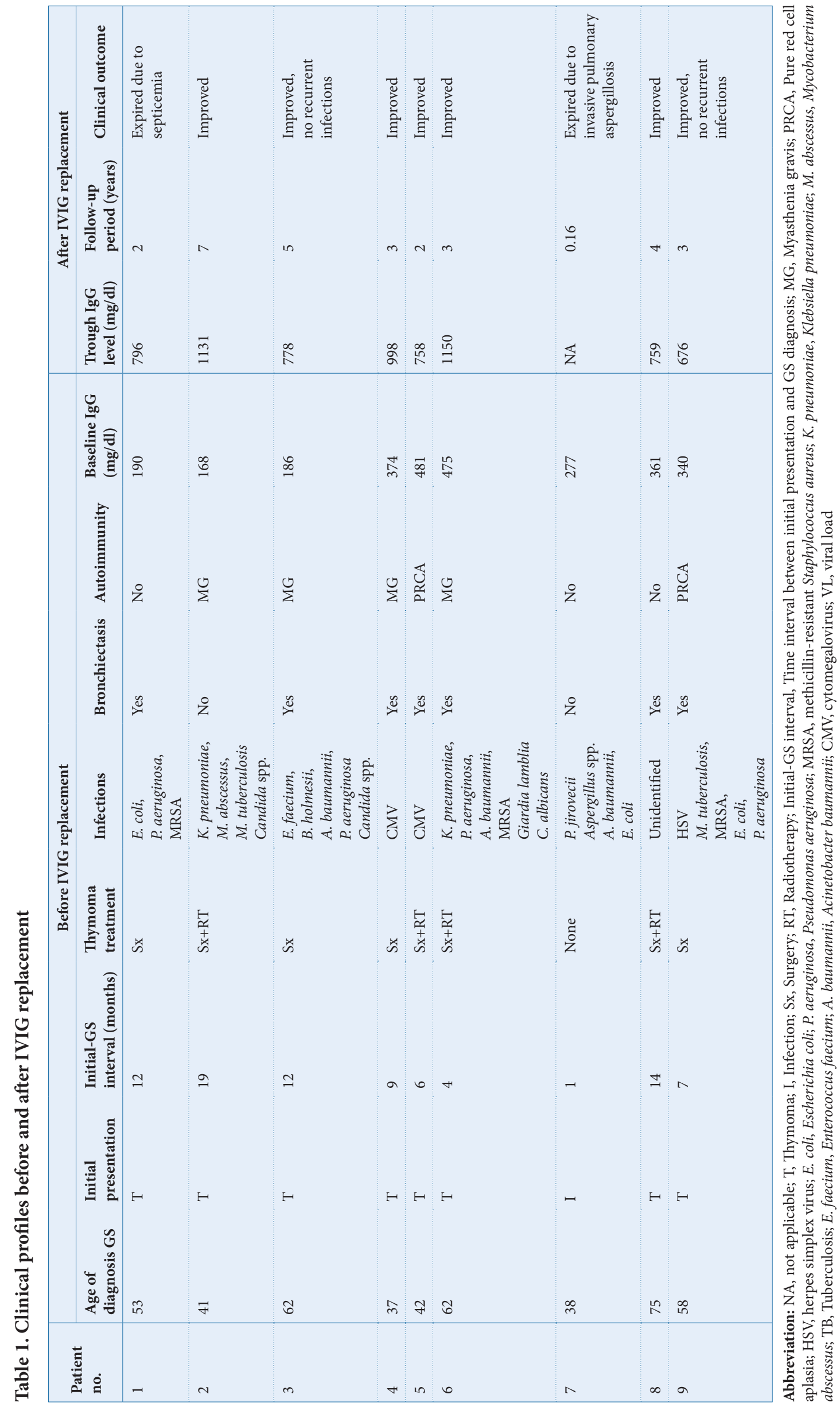




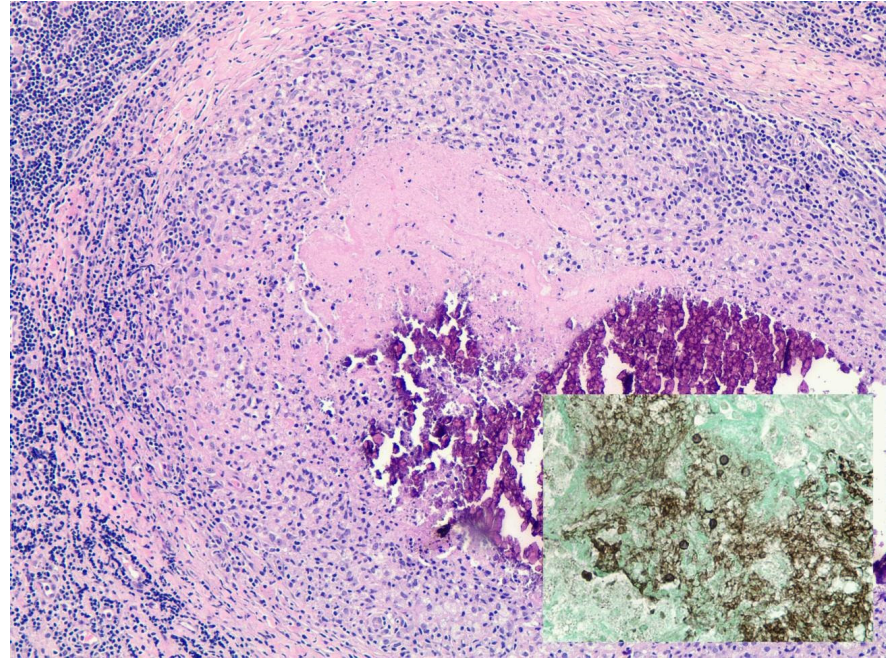

Figure 1. The lymph nodes in this patient showed numerous necrotizing granulomas with dystrophic calcification of the necrotic debris. The histiocytes in the lesion are not typical epithelioid histiocytes, suggesting the cellular mediated immunity defect. GMS stain reveals cup shape organisms compatible with Pneumocystis spp. (small inset)

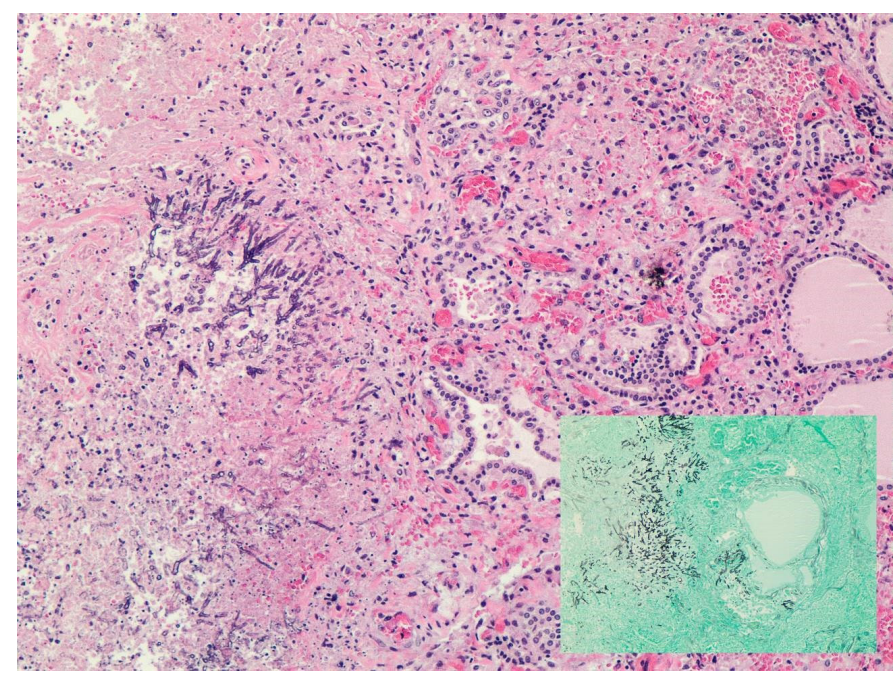

Figure 2. The thyroid gland was diffusely damaged by fungal invasion, resulting in extensive necrosis without granulomatous inflammation. The fungus is morphologically consistent with Aspergillus spp.

of the organism from infected cervical lymph node revealed $98 \%$ homology with $P$. jiroveci. Anti-IFN gamma autoantibody result was negative. He was then treated with IVIg replacement therapy and intravenous trimethoprim-sulfamethoxazole. He subsequently developed ventilator-associated pneumonia by Acinetobacter baumannii and expired due to overwhelming Escherichia coli septicemia shortly after the first dose of IVIg replacement. The autopsy finally revealed disseminated invasive aspergillosis distributing in lung, thyroid and brain tissues, as shown in figure 2.

Another patient (patient 4) presented with prolonged fever, progressively blurred vision and dyspnea on exertion. She was diagnosed with CMV retinitis and pneumonitis. CMV DNA 


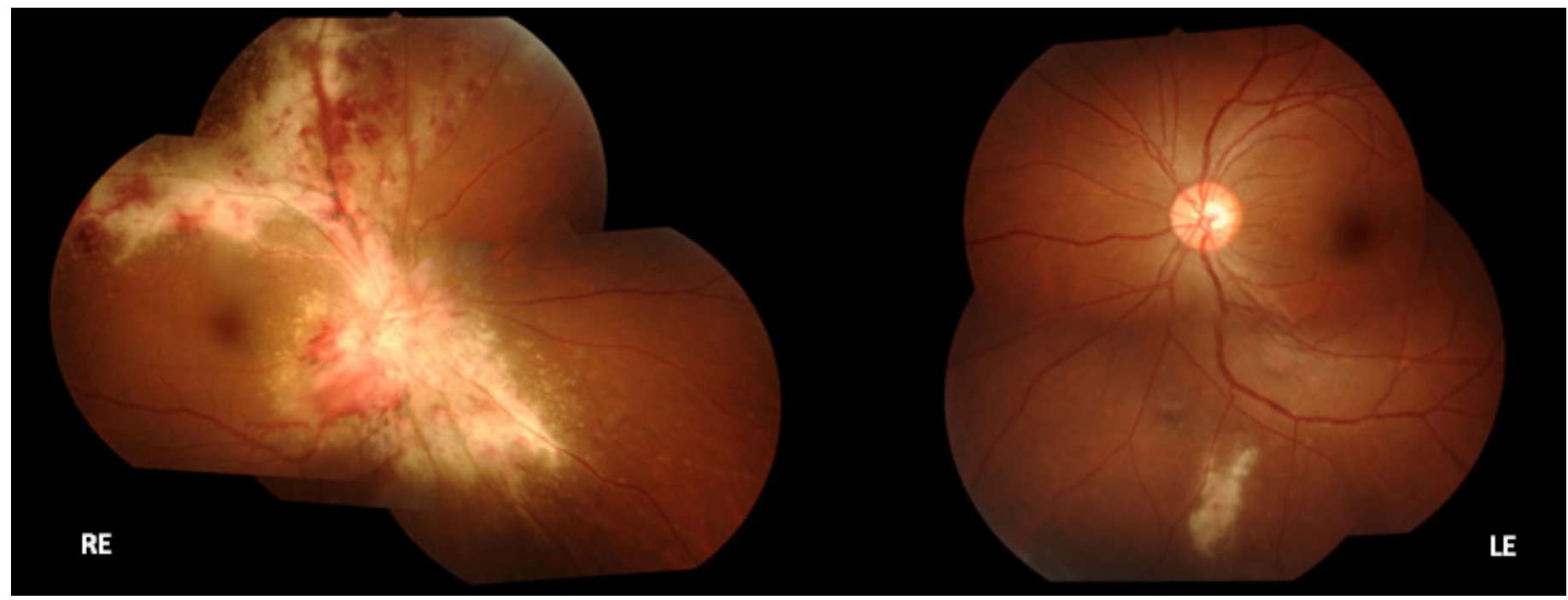

Figure 3. Bilateral cytomegalovirus retinitis in a 37 year-old female patient with Good syndrome. The right eye demonstrated classic necrotizing retinitis with cheese and ketchup appearance involving superior-temporal region, optic disc and adjacent retina resulting in subacute visual loss. A small area of retinitis with hemorrhage was also detected in the left eye without any symptom.

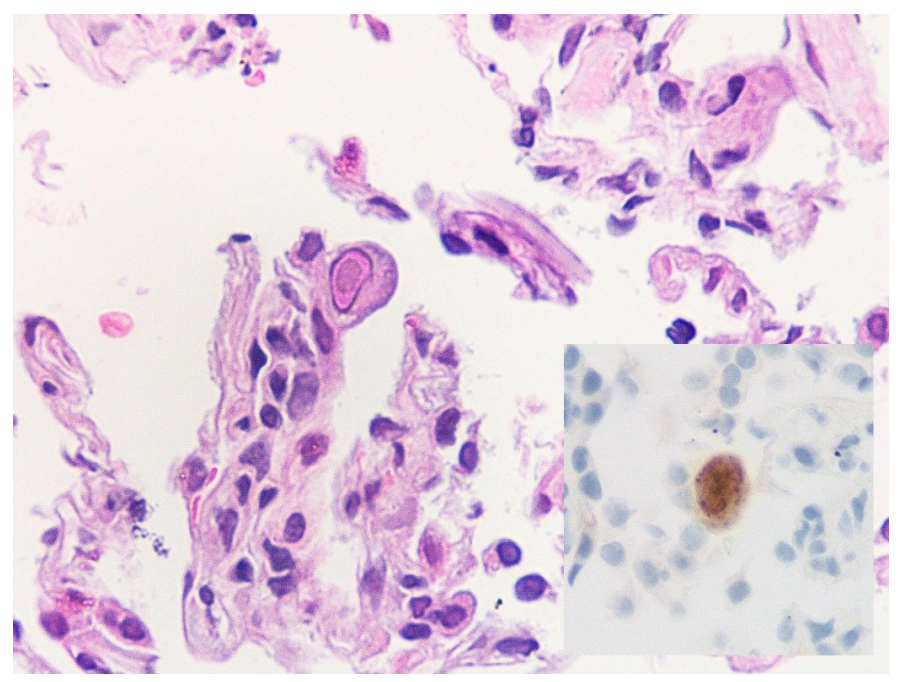

Figure 4. The patient lung tissue shows characteristic viral cytopathic change of the cytomegalovirus (CMV) infection. The infected cell shows enlarged nuclei with red-purple viral nuclear inclusion surrounded by eosinophilic ground glass halo. Immunohistochemistry (small inset) can highlight CMV antigen within the nucleus and cytoplasm of the infected cell.

was detected by polymerase chain reaction (PCR). Histopathology from transbronchial biopsy revealed cytopathic changes (figure 3, 4). She was treated with intravenous ganciclovir and IVIg replacement therapy. The overall clinical symptoms were improved which was concordant with the retinal findings during follow-up period.

\section{Discussion}

We illustrated nine cases of GS which were diagnosed between year 2005 and 2015. Two patients died due to infectious complications. The remaining seven cases continue monthly IVIg replacement. All patients in our study had been diagnosed with thymoma before detection of hypogammaglobulinemia.
In contrast, some patients in previous reports developed hypogammaglobulinemia before the diagnosis of thymoma. ${ }^{5}$ Interestingly, in a recent review, $82 \%$ of the patients had infection precede or simultaneously with the diagnosis of thymoma.? This could be explained by the under recognition of recurrent infections as the signs of phenocopies of primary immunodeficiency in adult patients with thymoma due to the rarity of GS in Thailand. On the contrary, Brown, LR, et al. reported that thymomas can be a subtle feature on chest $\mathrm{x}$-ray. A quarter of tumors were missed, leading to a diagnostic delay. ${ }^{9}$ Plain chest $\mathrm{X}$-ray shows merely a nonspecific broadened mediastinum, whereas, CT scan or magnetic resonance imaging of the chest have increased sensitivity for detection of thymoma and can also define the clinical stage of tumor. ${ }^{5,10}$ Therefore, CT of the chest should be considered for thymic mass detection in patient with unexplained hypogammaglobulinemia with highly suspicion of GS, but negative result from plain chest x-ray.

Previous study found that B lymphopenia occurred frequently, and that was more related to susceptibility of infections than was hypogammaglobulinemia. ${ }^{11}$ However, the level of $\mathrm{B}$ cells might be independent of hypogammaglobulinemia. ${ }^{12}$ Our finding was consistent with previous study that most patients (except patient 2) had very low to undetectable B-cell number. CMI defect was detected in all six cases according to CD4 lymphopenia or impaired T-cell function. Interestingly, all six patients with decreased number of NK cells had autoimmune diseases, including PRCA and MG. Previous report suggested that a reduction in NK cells may play a role in the development of autoimmune diseases. ${ }^{13}$ However, our study was too small and was not designed to conclude whether or not a decrease in NK cells is associated with autoimmune diseases. Additionally, the decrease in NK cells was reported associated with HSV infection. ${ }^{12}$ In our study, the patient 9 who had the lowest percent of NK cell also had chronic herpetic ulcer.

In our study, causative organisms in patients with pneumonia and/or sepsis were heterogeneous and partially different from previous study. ${ }^{5,6,8}$ The most common causative organism 
reported in a recent review was the encapsulated bacteria, such as Haemophilus influenzae and Streptococcus pneumoniae. ${ }^{7}$ Interestingly, seven of nine patients had already developed bronchiectasis confirmed by CT of the chest, which generally occurs after repeated episodes of infection. We hypothesized that our patients may have had an unrecognized lower respiratory tract infection with these encapsulated organisms for a lengthy period of time. Furthermore, a previous study also reported bronchiectasis in GS patients. ${ }^{8}$ In our study, CMV was the most common pathogen resulting in pneumonitis, retinitis, especially in patients who had either low $\mathrm{CD} 4$ number or impaired T-cell function. However, CMV infection still occurred in one patient (patient 4) who had normal CD4 number and T-cell function. A GS patient with normal CD4 number and $\mathrm{T}$ cell function who affected form CMV encephalitis had also been reported. ${ }^{2}$ This may be explained by the fact that both CMI and humoral immunity are necessary for controlling of CMV infection or the patient might have a reduction in specific $\mathrm{T}$ cell proliferative responses to $\mathrm{CMV}$ in the presence of a normal $\mathrm{T}$ cell proliferation to PHA., ${ }^{2,14}$

After regular IVIg replacement was administered, patients with previous CMV infection had no recurrences of CMV diseases which is consistent with a previous study in patients undergoing bone marrow transplantation showing that IVIg may prevent CMV infection. ${ }^{15}$ All patients in our study did not receive CMV-specific IgG. We described one patient with Pneumocystis jirovecii lymphadenitis and disseminated invasive aspergillosis, both of which are very rarely observed in non-HIV patients. We also found one patient with a history of chronic diarrhea without identifiable causes which was consistent with majority of cases from Tarr PE, et al. Our result found that thymectomy did not prevent or improve these autoimmune diseases, whereas IVIg replacement improved clinical control, which were consistent with a previous study. ${ }^{8}$ We observed that IVIg administration decreased the use of immunosuppressive drugs in four patients with MG. The occurrences of opportunistic infections in HIVseronegative patients should prompt physicians aggressively investigate for the occult immunodeficiency, especially in the setting of adult-onset. Apart from secondary immunodeficiency due to immunosuppression, malignancy, advanced age, metabolic diseases, malnutrition and disorder of protein loss, phenocopies of PID should be included in the differential diagnosis in such patients. The age of onset of PID varies depending on the defect. Incidence of PID is different in each age group, with the highest incidence rate among children below five years. ${ }^{16}$ However, PID is a diagnosis not only limited to children but also occur in adult. Recent reports of PID prevalence from the ESID and JMF revealed that $66 \%$ and $60 \%$ of patients were adult. ${ }^{17}$ One of the most common adult-onset CMI immunodeficiency in HIV-seronegative patients is anti-interferon gamma autoantibody. This disease shared similar patterns of opportunistic infections of advanced HIV but the pathogens were predominately non-tuberculous mycobacteria and usually associated with neutrophilic dermatosis. In contrast, patients with GS typically presented with wide spectrum of opportunistic infections, especially recurrent sinopulmonary tract infection and CMV diseases, while mycobacterial infection was scarcely reported even in the endemic area. $^{18}$

This study has some limitations. First, this is a retrospective study possibly resulting in missing or incomplete data. Second, the advance immunologic profiles such as anti-cytokine autoantibodies and response to vaccination had not been studied. Consequently, the mechanism of infections could not be thoroughly explained. Third, genetic studies such as whole exome or genome studies were not performed to identify the associated genetic defect. Forth, the patients enrolled in this study were from a single center. Therefore, our results may not be reflective of GS patients in other regions of Thailand and other countries.

In conclusion, clinical outcomes of patients with GS are more dependent on the severity of infections and associated hematologic, and autoimmune diseases than on the severity of thymoma itself. Early recognition and prompt IVIg replacement are crucially important and may change the natural course of this condition by keeping the patient infections free.

\section{Acknowledgements}

The authors gratefully acknowledge Voravich Leungwedchakarn, MD for assistance with clinical and laboratory services.

\section{Conflict of interest declaration}

The authors hereby declare no personal or professional conflicts of interest regarding any aspect of this study.

\section{Funding disclosure}

This was an unfunded study.

\section{References}

1. Good RA, Varco RL. A clinical and experimental study of agammaglobulinemia. J Lancet. 1955;75:245-71.

2. Kelleher P, Misbah SA. What is Good's syndrome? Immunological abnormalities in patients with thymoma. J Clin Pathol. 2003;56:12-6.

3. Agarwal S, Cunningham-Rundles C. Thymoma and immunodeficiency (Good syndrome): a report of 2 unusual cases and review of the literature. Ann Allergy Asthma Immunol. 2007;98:185-90.

4. Kampitak T, Putiworanat P, Chantaphakul H, Klaewsongkram J. Sclerosing cholangitis associated with good syndrome. J Investig Allergol Clin Immunol. 2009;19:509-10.

5. Kelesidis T, Yang O. Good's syndrome remains a mystery after 55 years: A systematic review of the scientific evidence. Clin Immunol. 2010;135: 347-63.

6. Malphettes M, Gerard L, Galicier L, Boutboul D, Asli B, Szalat R, et al. Good syndrome: an adult-onset immunodeficiency remarkable for its high incidence of invasive infections and autoimmune complications. Clin Infect Dis. 2015;61:e13-9.

7. Jansen A, van Deuren M, Miller J, Litzman J, de Gracia J, Saenz-Cuesta M, et al. Prognosis of Good syndrome: mortality and morbidity of thymoma associated immunodeficiency in perspective. Clin Immunol. 2016;171: 12-7.

8. Tarr PE, Sneller MC, Mechanic LJ, Economides A, Eger CM, Strober W, et al. Infections in patients with immunodeficiency with thymoma (Good syndrome). Report of 5 cases and review of the literature. Medicine (Baltimore). 2001;80:123-33.

9. Brown LR, Muhm JR, Gray JE. Radiographic detection of thymoma. AJR Am J Roentgenol. 1980;134:1181-8.

10. Yang WT, Lei KI, Metreweli C. Plain radiography and computed tomography of invasive thymomas: clinico-radiologic-pathologic correlation. Australas Radiol. 1997;41:118-24.

11. Montella L, Masci AM, Merkabaoui G, Perna F, Vitiello L, Racioppi L, et al. B-cell lymphopenia and hypogammaglobulinemia in thymoma patients. Ann Hematol. 2003;82:343-7. 
12. Martinez B, Browne SK. Good syndrome, bad problem. Front Oncol. 2014;4:307.

13. Mandal A, Viswanathan C. Natural killer cells: In health and disease. Hematol Oncol Stem Cell Ther. 2015;8:47-55.

14. Weber B, Braun W, Cinatl J, Jr., Doerr HW. Humoral immune response to human cytomegalovirus infection: diagnostic potential of immunoglobulin class and IgG subclass antibody response to human cytomegalovirus early and late antigens. Clin Investig. 1993;71:270-6.

15. Winston DJ, Ho WG, Lin CH, Bartoni K, Budinger MD, Gale RP, et al. Intravenous immune globulin for prevention of cytomegalovirus infection and interstitial pneumonia after bone marrow transplantation. Ann Intern Med. 1987;106:12-8.
16. Bousfiha AA, Jeddane L, Ailal F, Benhsaien I, Mahlaoui N, Casanova JL, et al. Primary immunodeficiency diseases worldwide: more common than generally thought. J Clin Immunol. 2013;33:1-7.

17. Rezaei N, Vries ED, Gambineri E, Haddad E. Common presentations and diagnostic approaches. In: Sullivan KE, Stiehm ER, editors. Stiehm's Immune Deficiencies. United States: Elsevier; 2014. p. 3-58.

18. Dong JP, Gao W, Teng GG, Tian Y, Wang HH. Characteristics of Good's Syndrome in China: A Systematic Review. Chin Med J (Engl). 2017;130: 1604-9. 